\title{
50 años de investigaciones de la Comunicación en México: un recuento descriptivo de la producción publicada*
}

\author{
Raúl Fuentes Navarro***
}

\section{Resumen}

Este trabajo presenta y contextualiza un recuento de publicaciones académicas producto de investigación de la Comunicación en México, durante los últimos 50 años, basado en el acervo documental del sitio ccdoc. El objetivo es analizar la producción científica del campo de la Comunicación. Al sistematizar e interpretar los datos, hay evidencias de un estado de "estancamiento concentrado", que hipotéticamente responde a una combinación de factores estructurales que habría que seguir investigando, preferentemente en proyectos comparativos con otras áreas científico-sociales y con la investigación de la Comunicación en otros países.

Palabras clave: Investigación de la Comunicación. Documentación académica. México.

\section{0 anos de pesquisa em Comunicação no México: um recorte descritivo da produção publicada \\ Resumo}

Este trabalho apresenta e contextualiza um recorte de publicações acadêmicas fruto de pesquisas na área da Comunicação no México, durante os últimos 50 anos, baseado no acervo documental do site ccdoc. O objetivo é analisar a produção científica da área da Comunicação. Ao sistematizar e interpretar os dados,

\footnotetext{
* Una versión previa fue presentada como ponencia en el X Congreso ALAIC (Bogotá, Colombia, septiembre 2010), en el GT Teoría y Metodología de la Investigación en Comunicación.

** Doctor en ciencias sociales y profesor-investigador del Departamento de Estudios Socioculturales y coor-dinador del Doctorado en Estudios CientíficoSociales del ITESO (Universidad Jesuita de Guadalajara). Miembro del Sistema Nacional de Investigadores (Nivel III) - CONACYT- y de la Academia Mexicana de Ciencias. Su principal línea de investigación ha sido la constitución del campo académico de la Comunicación. Autor de varios libros y artículos publicados en revistas naciones e internacionales. Ciudad do Mexico-DF, México. E-mail: raul@iteso.mx
} 
surgem evidências de um "estancamento concentrado", que hipoteticamente corresponde a uma combinação de fatores estruturais que deveriam continuar sendo investigadas, preferencialmente em projetos comparativos com outras áreas sócio-científicas e com a pesquisa na área da Comunicação em outros países.

Palavras-chave: Pesquisa em Comunicação. Documentação acadêmica. México.

\section{0 years of research in Communication area in Mexico: some descriptive data of the published production \\ Abstract}

Counting and contextualizing some data on Mexican Communication Research published academic production over five decades, extracted from the website $c c d o c$. The objective is to analyze the scientific production of the Communication area. Some evidences interpreted by the author emerge to sustain a hypothesis concerning the "concentrated stagnation" which seems to characterize the field, and which leads to the recognition of a combination of structural factors, that suggest further research efforts, especially in comparative projects with other scientific-social fields and with other countries.

Keywords: Communication Research. Scholarly Documentation. Mexico.

\section{Introducción}

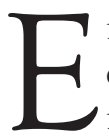

n 2010, en México se conmemoran 50 años de la existencia de programas académicos dedicados al estudio de la Comunicación. Si bien antes de 1960 se habían fundado licenciaturas universitarias en periodismo (en la Escuela de Periodismo Carlos Septién García, en la Universidad Nacional Autónoma de México y en la Universidad Veracruzana), se ha convenido en el campo académico nacional el reconocimiento a la licenciatura en (Ciencias de la) Comunicación de la Universidad Iberoamericana como la fundadora y pionera de un proyecto académico que, al paso de los años, resultó ser el predominante en México y América Latina, al grado que dos de las tres escuelas de periodismo que la precedieron en el país, cambiaron su orientación hacia las "Ciencias de la Comunicación” en los años setenta ${ }^{1}$. Es anecdótico que,

\footnotetext{
${ }^{1}$ Recientemente, la colega chilena Claudia Mellado (2010a, 2010b) ha explorado de una manera muy interesante las consecuencias y secuelas académicas y profesionales de la indistinción entre "periodismo" y "Comunicación" en los países latinoamericanos.
} 
poco después de su fundación, el programa de la Iberoamericana debió cambiar su nombre por el de "Ciencias y Técnicas de la Información”, debido a que según las autoridades nacionales, su objeto no estaba relacionado con telégrafos o carreteras. Una vez que la denominación original se había establecido en México y en otros países, la Iberoamericana lo recuperó en los años setenta, aunque prescindiendo del término "Ciencias".

No obstante, en las escuelas de Comunicación mexicanas se comenzaron a incorporar actividades de investigación sistemática apenas a mediados de los años 1970, debido quizá al carácter novedoso y académicamente indeterminado de los programas de formación profesional, cuya demanda en cinco décadas no ha dejado de crecer. Según datos publicados por FELAFACS² (2009), hay más de un millar de programas de "Comunicación" operando en el país, la mayoría de ellos en cientos de instituciones de carácter marcadamente mercantil. Y sin embargo, apenas se cuenta con unos cincuenta programas de posgrado, de los cuales sólo ocho han alcanzado la acreditación oficial "de calidad" académica, y únicamente cinco se consideran "consolidados"3. La proporción de más de 75 mil estudiantes de licenciatura y menos de mil de posgrado en Comunicación en las universidades mexicanas, ha generado la fórmula diagnóstica de un campo académico hipertrofiado en sus estructuras de pregrado y subdesarrollado en sus instancias de investigación y profesionalización avanzada.

De manera que la investigación académica ("científica”) de la Comunicación en México cuenta apenas con unos 25 años de trayectoria institucional reconocible, la mitad del tiempo que los estudios universitarios ("profesionalizantes") sobre la Comunicación, con los que mantiene una relación tensa, incierta y fragmentada.

\footnotetext{
${ }^{2}$ Federación Latinoamericana de Facultades de Comunicación Social.

${ }^{3}$ Para una consideración analíticamente más detallada de este contexto institucional, ver Fuentes (2010a; 2010b). Los cinco programas de maestría en Comunicación "consolidados" son los ofrecidos por la Universidad Nacional (UNAM), la Universidad Iberoamericana (UIA), la Universidad de Guadalajara (UdeG), el ITESO y el Tecnológico de Monterrey (ITESM). No hay programas de doctorado en Comunicación acreditados, pero se incluyen áreas de concentración en al menos seis doctorados en ciencias sociales que cuentan con la acreditación.
} 
En el Sistema Nacional de Investigadores (SNI), aproximadamente 150 practicantes de esta especialidad (o "subdisciplina", catalogada como tal bajo el rubro "Sociología"), cuentan actualmente con el reconocimiento oficial en los diversos niveles de que consta el Sistema, cantidad que es apreciable pues equivale aproximadamente al $1 \%$ de los investigadores nacionales.

En este trabajo, con el objetivo es analizar la producción científica del campo de la Comunicación, se exponen y sistematizan algunos datos representativos de la "producción académica" de investigaciones de la Comunicación en México, con la intención de sustentar algunas interpretaciones sobre la institucionalización de esta actividad en el país en una perspectiva temporal. Se utiliza para ello un recurso documental (el sitio Documentación en Ciencias de la Comunicación, ccdoc) ${ }^{4}$ de utilidad imprescindible para el seguimiento evaluativo de esta producción, y para la realización de análisis comparativos tanto "internos" (entre instituciones, subcampos temáticos, épocas, investigadores...) como "externos" (con otras áreas de investigación, con otros países...) (Fuentes, 2005).

\section{Los productos de investigación registrados en ccdoc}

Resultado de un proyecto que permitió aprovechar avances tecnológicos de la era digital para los fines de documentación académica en el campo de la Comunicación en México, que habían orientado los esfuerzos del autor desde 1983, en el sitio ccdoc se han recuperado las referencias bibliográficas sistematizadas en tres libros (publicados en 1988, 1996 y 2003), lo que ha aportado al menos tres ventajas con respecto a sus antecedentes: en primer lugar, la disponibilidad en línea para la consulta, pues se trata de un sitio "abierto"; en segundo lugar, la actualización, pues las referencias pueden incorporarse continuamente al acervo; y en tercer lugar, el acceso a los textos completos digitalizados de todos los documentos

\footnotetext{
${ }^{4}$ [http://ccdoc.iteso.mx], disponible en línea desde octubre de 2003, incorporado a la Iniciativa de Archivos Abiertos (OAI), gracias a un proyecto coordinado por el autor desde el Departamento de Estudios Socioculturales del ITESO, con el apoyo del Consejo Nacional de Ciencia y Tecnología (CONACYT). Para una exposición detallada del proyecto, Fuentes (2005).
} 
cuya reproducción es autorizada por los poseedores de los derechos respectivos. Dotado de las tecnologías de búsqueda más avanzadas, el sitio (apoyado operativamente por la Biblioteca "Dr. Jorge Villalobos Padilla SJ" del ITESO) cuenta en julio de 2010 con más de 5200 documentos referidos, aproximadamente el 40\% de los cuales pueden "descargarse" en texto completo (formato .pdf).

El proyecto de documentación circunscribe el acervo a productos publicados de investigación sobre la Comunicación en México: libros, capítulos, artículos y tesis de posgrado. El documento más antiguo es un artículo publicado en 1956, aunque por supuesto la investigación en ciertas áreas temáticas, como la historia del periodismo, cuenta en el país con antecedentes previos a esa fecha, así sean escasos. Otra delimitación es el énfasis en la producción académica, en detrimento de la realizada por agencias no universitarias, cuyos resultados no suelen ser publicados.

Para este trabajo se ha seleccionado un corpus compuesto por libros, capítulos y artículos (excluyendo tesis de posgrado, que han sido analizadas con otros parámetros en Fuentes, 2010a), generados por los investigadores adscritos a universidades mexicanas (excluyendo los publicados por extranjeros sobre la Comunicación mexicana), entre 1960 y 2009. Como se desprende de los datos contenidos en el Cuadro No. 1, durante los primeros 25 de los 50 años considerados, se publicó el $12.5 \%$ de los productos y, en consecuencia, el $87.5 \%$ restante entre 1985 y 2009. Por ello puede afirmarse que la institucionalización de esta actividad está apenas cursando su tercera década.

También es necesario tomar en cuenta para contextualizar los datos que, como organizaciones trans-institucionales, el Consejo Nacional para la Enseñanza y la Investigación de las Ciencias de la Comunicación (CONEICC) fue constituido en 1976 y ha celebrado quince encuentros nacionales entre 1982 y 2010, y la Asociación Mexicana de Investigadores de la Comunicación (AMIC), constituida en 1979, ha organizado 22 encuentros entre 1980 y 2010. Ambas organizaciones han mantenido la publicación regular de anuarios de investigación (CONEICC desde 1994 y AMIC desde 2001), de donde surgen, respectivamente 217 y 120 de los artículos y capítulos contenidos en el corpus de este análisis. 
Cuadro n. 1: Productos publicados de la investigación de la Comunicación en, por formato y lustro, 1960-2009 (ccdoc)

\begin{tabular}{l|l|l|l|l}
\hline Lustro & Libros & Capítulos & Artículos & Total \\
\hline $1960-1964$ & 0 & 0 & 2 & $2(0.0 \%)$ \\
\hline $1965-1969$ & 11 & 1 & 1 & $13(0.3 \%)$ \\
\hline $1970-1974$ & 15 & 0 & 34 & $49(1.1 \%)$ \\
\hline $1975-1979$ & 44 & 7 & 121 & $172(3.8 \%)$ \\
\hline $1980-1984$ & 117 & 38 & 173 & $328(7.3 \%)$ \\
\hline $1985-1989$ & 102 & 136 & 239 & $477(10.7 \%)$ \\
\hline $1990-1994$ & 114 & 199 & 419 & $732(16.4 \%)$ \\
\hline $1995-1999$ & 103 & 267 & 531 & $901(20.2 \%)$ \\
\hline $2000-2004$ & 134 & 453 & 480 & $1067(23.9 \%)$ \\
\hline $2005-2009$ & 78 & 263 & 388 & $729(16.3 \%)$ \\
\hline Totales & $718(16.1 \%)$ & $1364(30.5 \%)$ & $2388(53.4 \%)$ & $4470(100 \%)$ \\
\hline
\end{tabular}

Al revisar la producción, es claro que el proceso de constitución del campo de la investigación académica de la Comunicación en México comenzó a mediados de los años setenta, cuando se abrieron también los primeros programas de maestría en la Zona Metropolitana de la Ciudad de México (UIA y UNAM). Si bien los esfuerzos originales se orientaron por modelos de la investigación empírica estadounidense, la mayor parte de la producción se enmarcó más bien en la tradición “crítica” latinoamericana y europea. Pero desde mediados de los años ochenta, la investigación y los posgrados dieron indicios de la prevalencia de otros patrones de desarrollo, quizá menos definidos pero más diversificados, sobre los cuales el campo académico alcanzó algún grado de crecimiento y consolidación.

Esta reestructuración se ha explicado (Fuentes, 1998) por la incorporación de un tipo de investigadores que buscaron reintegrar el rigor científico y el anclaje empírico con el sentido crítico y el compromiso social en proyectos de investigación institucional e intelectualmente independientes de la docencia en licenciatura, y por tanto no comprometidas con una "disciplina de la Comunicación” autónoma como se había venido entendiendo, sino con abordajes diversos pero más amplios, dentro de las ciencias sociales y de la cultura. Sobre estas bases, durante la segunda mi- 
tad de los ochenta se crearon nuevos centros de investigación en algunas universidades, especialmente la Universidad de Colima, la de Guadalajara y la Iberoamericana, donde encontraron apoyo investigadores formados en doctorados en ciencias sociales del país o el extranjero, con perfiles reconocibles por las instancias oficiales de impulso a la investigación científica, y con vocación para una nueva vinculación docente, más orientada hacia el posgrado que hacia las licenciaturas.

El cambio en las políticas oficiales para la educación superior y la investigación científica, algunos impulsos de descentralización geográfica, y la incorporación de nuevas perspectivas para el estudio de la Comunicación, con énfasis sobre el desarrollo metodológico y teórico crítico y el sustento empírico de las formulaciones en marcos de ciencia social, coincidieron así en las últimas dos décadas del siglo XX como factores de consolidación del campo académico, que pareció haber estabilizado temporalmente la lucha por las "posiciones" de liderazgo, intensa en el origen, en un modelo de colaboración e intercambio interinstitucional y entre un grupo reducido de individuos, de búsqueda de la legitimación colectiva ante diversos sectores de la investigación en ciencias sociales.

Pero al mismo tiempo, esta "estabilización" de la lucha por las posiciones en el campo tuvo que ver también con el deterioro de las condiciones institucionales y la pugna, al interior de los establecimientos universitarios, por los espacios y recursos requeridos para el desarrollo académico. En este sentido, la investigación y la formación en posgrado han debido sostener desde entonces una lucha contra la "burocratización", la "racionalización” y la priorización de una docencia instrumentalista en la mayor parte de las universidades. La consolidación académica del campo de la Comunicación ha estado condicionada, de esta manera, por la propia modernización "neo-liberal” y los reajustes presupuestales de las universidades (sobre todo las públicas) ante las "nuevas" crisis económicas del país.

En la década más reciente han mantenido su vigencia las principales condiciones estructurales determinantes del contradictorio desarrollo de la investigación académica de la Comunicación en las 
décadas anteriores ${ }^{5}$, aunque algunas de estas condiciones se han agravado. Habría que considerar, entre otros factores, que los procesos políticos asociados con el "cambio de régimen" o la alternancia partidaria en la presidencia de la república a partir del año 2000, han generado cambios discursivos y estructurales en todos los órdenes, incluyendo la Comunicación, difíciles de evaluar, pero poco consistentes en todo caso con la imagen "democratizadora" que se podía tener de ellos antes del año 2000. Podría sostenerse incluso que la creciente atención a los sistemas y procesos "de Comunicación" en los debates públicos y de interés general, ha implicado un simultáneo desdibujamiento conceptual en los marcos desde los cuales los agentes sociales especializados en la operación, y en la investigación científica, de la multidimensional operación social de los medios de difusión masiva intervienen en ella. La "instrumentalización" reduccionista de la Comunicación ha permeado también, sin duda y sin que debiera causar sorpresa, a las universidades.

En la dimensión institucional, contexto de las estrategias para enfrentar los cambios en los entornos sociocultural y cognoscitivo, pueden también percibirse tensiones recientes: la investigación académica de la Comunicación ya establecida se ha fortalecido, y en la última década ha dado muestras de renovación generacional, casi imperceptibles hasta mediados de los noventa. Pero en relación con el expansivo crecimiento de la oferta de programas de licenciatura en Comunicación, se ha mantenido en un estado precario de desarrollo, equivalente en varios de sus indicadores al

\footnotetext{
${ }^{5}$ El estudio de Fuentes (1998) elaboró tres dimensiones como contextos relevantes de la estructuración del campo: la cognoscitiva, la sociocultural y la institucional. En la primera se incluyeron "factores y procesos de cambio que han afectado la producción, reproducción y circulación del conocimiento sobre la Comunicación y las estructuras y fenómenos socioculturales en términos más amplios, así como el sentido de las actividades y sistemas académicos, científicos, universitarios, tanto desde el 'exterior' como al 'interior' del campo académico de la Comunicación”. En la dimensión sociocultural se consideraron a su vez "factores y procesos de cambio ocurridos en esas actividades y sistemas en relación con las estructuras (nacionales e internacionales) culturales, políticas y económicas, sujetas a transformaciones de amplio alcance en los años recientes". Finalmente, "la dimensión institucional refiere concreta y específicamente a los cambios en que confluyen los factores 'cognoscitivos' y los 'socioculturales' tanto al interior del sistema nacional de educación superior como en las relaciones universidad-sociedad” (Fuentes, 1998, p.48).
} 
de la década anterior, como el correspondiente a los programas de posgrado acreditados, e incluso inferior, como el número de centros de investigación activos o de revistas académicas.

En el Cuadro n. 1 puede apreciarse una "disminución” en el número de productos registrados para el lustro más reciente (20052009), menor incluso al de 1990-1994. Una parte de esa reducción puede reflejar un subregistro en ccdoc, debido a la demora en la aparición o la captura de las publicaciones, pero también habría que considerar la proliferación de publicaciones en medios electrónicos (no incluidas en el corpus) y el hipotético descenso de la producción en sí, debido al deterioro de las condiciones generales.

De cualquier manera, es claro que ha aumentado la fragmentación temática y metodológica de la investigación, y aunque se han establecido dinámicas de investigación en universidades donde no las había en décadas anteriores, casi el 70\% de la producción sigue generándose en solo siete instituciones, donde residen los programas de posgrado acreditados y en donde están adscritos la mayor parte de los investigadores reconocidos por el SNI. De ahí que la fórmula diagnóstica que mejor describe el estado presente del campo de la investigación académica de la Comunicación en México sea el de un "estancamiento concentrado".

\section{El origen institucional de los productos de investigación}

En el Cuadro No. 2 se desglosan los productos de investigación registrados en ccdoc, según la adscripción institucional de sus autores. Cabe hacer notar que la actividad académica en el campo de la Comunicación comenzó en diversas épocas para cada universidad: en la UNAM desde la década de los cincuenta, en la UIA y el ITESO en los sesenta, en la UAM y el ITESM en los setenta, y en la UdeG y la Universidad de Colima en los ochenta. Muchas de las instituciones que iniciaron más recientemente sus actividades de producción en este campo ${ }^{6}$, así como algunas que las suspendieron o

\footnotetext{
${ }^{6}$ Entre las que se pueden destacar la Universidad de Las Américas-Puebla (UDLAP), la Universidad Autónoma del Estado de México (UAEM), la Universidad Autónoma de Nuevo León (UANL), la Universidad de Sonora (UNISON), la Universidad Autónoma de la Ciudad de México (UACM), la Universidad Intercontinental (UIC) y la Universidad Autónoma de Baja California (UABC).
} 
redujeron en décadas pasadas ${ }^{7}$, cuentan en la columna de "Otras", al no alcanzar un mínimo de 100 publicaciones en el periodo total. Una aclaración adicional es necesaria, dada la diferente escala y carácter de las instituciones: para la UNAM, la UAM, la UdeG y la UCOL, instituciones públicas, se considera la producción proveniente de diversas unidades y sedes académicas (facultades, centros, institutos, escuelas) donde se adscriben investigadores de la Comunicación. Para la UIA y el ITESM, sistemas particulares con sedes en diferentes ciudades, igualmente se suma la producción de todas ellas. Sólo el ITESO contiene exclusivamente la producción proveniente de su actual Departamento de Estudios Socioculturales (y sus precedentes organizacionales: el Departamento de Comunicación, la Maestría en Comunicación y la Escuela de Ciencias de la Comunicación).

Cuadro n. 2: Productos publicados de la investigación de la Comunicación en México, por universidad y lustro, 1960-2009 (ccdoc)

\begin{tabular}{l|l|l|l|l|l|l|l|l|l}
\hline Lustro & UNAM & UAM & UdeG & UIA & ITESO & UCOL & ITESM & Otras & Total \\
\hline $1960-64$ & 1 & & & & & & & 1 & 2 \\
\hline $1965-69$ & 7 & & & & & & & 6 & 13 \\
\hline $1970-74$ & 36 & & & & & & & 13 & 49 \\
\hline $1975-79$ & 69 & 10 & & 4 & 3 & & & 86 & 172 \\
\hline $1980-84$ & 87 & 77 & 4 & 20 & 29 & & 1 & 110 & 328 \\
\hline $1985-89$ & 105 & 81 & 55 & 49 & 48 & 34 & 0 & 105 & 477 \\
\hline $1990-94$ & 113 & 145 & 92 & 118 & 80 & 44 & 11 & 129 & 732 \\
\hline $1995-99$ & 123 & 125 & 118 & 155 & 93 & 54 & 40 & 193 & 901 \\
\hline $2000-04$ & 140 & 113 & 147 & 108 & 89 & 37 & 60 & 373 & 1067 \\
\hline $2005-09$ & 74 & 95 & 80 & 33 & 41 & 18 & 42 & 346 & 729 \\
\hline Totales & 755 & 646 & 496 & 487 & 383 & 187 & 154 & 1362 & 4470 \\
& $16.8 \%$ & $14.5 \%$ & $11.1 \%$ & $10.9 \%$ & $8.6 \%$ & $4.2 \%$ & $3.4 \%$ & $30.5 \%$ & $100 \%$ \\
\hline
\end{tabular}

Vale reiterar que los datos consignados en el Cuadro No. 2 corresponden a la producción de los productos de investigación, según la adscripción institucional de sus autores. Para cada una de las universidades hay también una distribución interna, variable por lustros, pero que mantiene autores constantes a lo largo del tiempo: precisamente los investigadores de la Comunicación más

${ }^{7}$ Como la Universidad Anáhuac, el Instituto Latinoamericano de Estudios Transnacionales (ILET) o el Centro de Estudios Económicos y Sociales del Tercer Mundo (CEESTEM). 
"productivos" (según el número de sus publicaciones) del país. En el corpus destacan, de cada una de las universidades, los individuos enlistados en el Cuadro No. 3. No es sorprendente que sean también, estos investigadores, los que han alcanzado los niveles más altos de reconocimiento en el Sistema Nacional de Investigadores y el mayor prestigio internacional. La mayoría de ellos y ellas han estado publicando productos de investigación desde hace veinticinco años o más.

\section{Cuadro n. 3 - Investigadores con mayor número de publicaciones, por institución de adscripción, 1960-2009 (ccdoc)}

\begin{tabular}{|c|c|c|c|c|c|}
\hline Universidad & Investigador(a) & Libros & Caps. & Arts. & Total \\
\hline \multirow{3}{*}{ UNAM } & Delia Ma. Crovi Druetta (FCPyS) & 12 & 31 & 40 & 83 \\
\hline & Raúl Trejo Delarbre (IIS) & 16 & 22 & 15 & 53 \\
\hline & Fátima Fernández Christlieb (FCPyS) & 7 & 15 & 29 & 51 \\
\hline \multirow{3}{*}{ UAM } & Javier Esteinou Madrid (Xochimilco) & 24 & 36 & 113 & 173 \\
\hline & Néstor García Canclini (Iztapalapa) a & 19 & 25 & 16 & 60 \\
\hline & Beatriz Solís Leree (Xochimilco) & 5 & 6 & 13 & 24 \\
\hline \multirow{3}{*}{ UdeG } & Enrique E. Sánchez Ruiz (DECS) & 14 & 33 & 70 & 117 \\
\hline & Guillermo Orozco Gómez (DECS) & 12 & 21 & 34 & 67 \\
\hline & $\begin{array}{l}\text { Francisco de J. Aceves González } \\
\text { (DECS) }\end{array}$ & 4 & 12 & 32 & 48 \\
\hline \multirow{3}{*}{ UIA } & Guillermo Orozco Gómez (Santa Fe) b & 9 & 18 & 29 & 56 \\
\hline & Héctor Gómez Vargas (León) & 9 & 5 & 29 & 43 \\
\hline & Claudia Benassini Félix $\left(\right.$ Santa Fe) ${ }^{c}$ & 4 & 4 & 24 & 32 \\
\hline \multirow{3}{*}{ ITESO } & Raúl Fuentes Navarro (DESO) & 19 & 38 & 91 & 148 \\
\hline & Rossana Reguillo Cruz (DESO) & 11 & 31 & 72 & 114 \\
\hline & Cristina Romo Gil (DESO) & 5 & 6 & 11 & 22 \\
\hline \multirow{3}{*}{ UCOL } & Luis Jesús Galindo Cáceres (CUIS) d & 16 & 15 & 43 & 74 \\
\hline & Jorge A. González Sánchez (CUIS) e & 7 & 12 & 27 & 46 \\
\hline & Ana B. Uribe Alvarado (CUIS) & 2 & 3 & 9 & 14 \\
\hline \multirow{3}{*}{ ITESM } & María de la Luz Casas Pérez (Morelos) & 1 & 12 & 25 & 38 \\
\hline & Octavio Islas Carmona (Edomex) & 3 & 6 & 29 & 38 \\
\hline & $\begin{array}{l}\text { José Carlos Lozano Rendón } \\
\text { (Monterrey) }\end{array}$ & 1 & 8 & 20 & 29 \\
\hline
\end{tabular}

NOTAS/ a: Recuento parcial de sus publicaciones

b: Actualmente en el DECS UdeG

c: Actualmente en el ITESM

$\mathrm{d}$ : Actualmente en la U. Intercontinental

e: Actualmente en el CIICH UNAM 
Los datos correspondientes a la "producción" de libros, capítulos y artículos pueden también ilustrar otro aspecto del "estancamiento" concentrado del campo de la investigación académica de la Comunicación en México: el de la "edición", base institucional de la circulación de los productos científicos, en el cual tienen una participación destacada las mismas universidades. En el Cuadro No. 4 se cruzan los datos de edición del corpus analizado, por institución, en tanto productoras y editoras de los textos.

Cuadro n. 4: Productos de investigación de la Comunicación en México, por universidad productora y editora, 1960-2009 (ccdoc)

\begin{tabular}{|c|c|c|c|c|c|c|c|c|c|}
\hline Editora & UNAM & UAM & UdeG & UIA & ITESO & UCOL & ITESM & Otras & Total \\
\hline \multicolumn{10}{|l|}{ Productora } \\
\hline UNAM & $\begin{array}{l}280 \\
37.1 \% \\
\end{array}$ & 31 & 35 & 12 & 4 & 14 & 3 & 376 & $\begin{array}{l}755 \\
16.8 \% \\
\end{array}$ \\
\hline UAM & 26 & $\begin{array}{l}204 \\
31.6 \% \\
\end{array}$ & 33 & 33 & 4 & 16 & 2 & 328 & $\begin{array}{l}646 \\
14.5 \% \\
\end{array}$ \\
\hline UdeG & 10 & 7 & $\begin{array}{l}256 \\
51.6 \% \\
\end{array}$ & 8 & 12 & 7 & 3 & 193 & $\begin{array}{l}496 \\
11.1 \% \\
\end{array}$ \\
\hline UIA & 4 & 13 & 17 & $\begin{array}{l}209 \\
42.9 \% \\
\end{array}$ & 5 & 24 & 2 & 214 & $\begin{array}{l}487 \\
10.9 \% \\
\end{array}$ \\
\hline ITESO & 8 & 11 & 31 & 7 & $\begin{array}{l}132 \\
34.5 \% \\
\end{array}$ & 8 & 3 & 183 & $\begin{array}{l}383 \\
8.6 \% \\
\end{array}$ \\
\hline UCOL & 1 & 2 & 19 & 15 & 12 & $\begin{array}{l}84 \\
44.9 \% \\
\end{array}$ & 0 & 54 & $\begin{array}{l}187 \\
4.2 \% \\
\end{array}$ \\
\hline ITESM & 7 & 4 & 14 & 8 & 0 & 2 & $\begin{array}{l}14 \\
9.1 \% \\
\end{array}$ & 105 & $\begin{array}{l}154 \\
3.4 \% \\
\end{array}$ \\
\hline Otras & 100 & 109 & 112 & 77 & 13 & 76 & 27 & $\begin{array}{l}847 \\
62.3 \%\end{array}$ & $\begin{array}{l}1362 \\
30.5 \%\end{array}$ \\
\hline Total & $\begin{array}{l}436 \\
9.8 \% \\
\end{array}$ & $\begin{array}{l}381 \\
8.5 \% \\
\end{array}$ & $\begin{array}{l}517 \\
11.6 \% \\
\end{array}$ & $\begin{array}{l}369 \\
8.2 \% \\
\end{array}$ & $\begin{array}{l}182 \\
4.1 \% \\
\end{array}$ & $\begin{array}{l}231 \\
5.2 \% \\
\end{array}$ & $\begin{array}{l}54 \\
1.2 \% \\
\end{array}$ & $\begin{array}{l}2300 \\
51.4 \%\end{array}$ & $\begin{array}{l}4470 \\
100 \% \\
\end{array}$ \\
\hline
\end{tabular}

De acuerdo con los datos del Cuadro No. 4, hay una tendencia variable por universidad para editar los productos de investigación producidos por sus propios investigadores: en el extremo "endogámico”, un poco más de la mitad de los textos generados por la Universidad de Guadalajara se publicaron por la misma institución, mientras que en el otro extremo, apenas el $9 \%$ de los producidos en el ITESM aparecen en ediciones con su sello. No obstante, esta especie de "índice de endogamia" alcanza el 40\% de promedio para las siete instituciones, porcentaje muy alto si se consideran las implicaciones que, por ejemplo, esta práctica tiene para el arbitraje 
“doble-ciego" de los textos. Correspondientemente, en las siete instituciones se han publicado productos de investigación provenientes de las otras seis (con dos excepciones: de la Universidad de Colima en el ITESM, y del ITESM en el ITESO), aunque en proporciones mínimas.

Pero el dato probablemente más significativo sea que más del $62 \%$ de la producción de las instituciones identificadas como "Otras", diferentes a las siete universidades más productivas, ha sido publicado también por "otras" instituciones, porcentaje un poco mayor al de la producción que sí es incorporado por las primeras, en proporciones variables. Por una parte, se revela así que prácticamente la mitad (48.6\%) de los productos de investigación han sido publicados por las mismas instituciones donde se genera el $69.5 \%$ de ellos, lo cual corrobora la alta "concentración" del campo. Por otra parte, conviene desglosar un poco el $51.4 \%$ de la edición por "otras" instituciones, que equivale a 2300 textos, porque en este caso la categoría "Otras" incluye tipos diversos de instituciones, señaladamente tres: las editoriales comerciales; las instituciones que son solamente editoras (como el CONEICC, la AMIC o la Fundación Manuel Buendía) y no productoras, además de las otras universidades; y las instituciones extranjeras (tanto editoras como editoras/productoras). En el Cuadro n. 5 se presentan los resultados de este desglose, por institución productora.

Cuadro n. 5: Productos de investigación de la Comunicación en México, por universidad productora, publicados por "otras" editoras, 1960-2009 (ccdoc)

\begin{tabular}{l|l|l|l|l}
\hline Editoras & Comerciales & $\begin{array}{l}\text { No-productoras } \\
\text { y otras univ. }\end{array}$ & Extranjeras & Total \\
\hline Productora & & & & \\
\hline UNAM & 80 & 253 & 43 & 376 \\
\hline UAM & 54 & 184 & 90 & 328 \\
\hline UdeG & 3 & 99 & 91 & 193 \\
\hline UIA & 45 & 125 & 44 & 214 \\
\hline ITESO & 9 & 55 & 119 & 183 \\
\hline UCOL & 8 & 22 & 24 & 54 \\
\hline ITESM & 5 & 72 & 28 & 105 \\
\hline Otras & 144 & 631 & 72 & 847 \\
\hline Total parcial & $348(15.1 \%)$ & $1441(62.6 \%)$ & $511(22.3 \%)$ & $2300(100 \%)$ \\
\hline$\%$ del corpus & $(7.8 \%)$ & $(32.2 \%)$ & $(11.4 \%)$ & $(51.4 \%)$ \\
\hline
\end{tabular}




\section{Implicaciones para análisis posteriores}

De los datos del Cuadro No. 5 se desprende una constatación interesante: la "edición", y por lo tanto la circulación de los productos de la investigación mexicana sobre la Comunicación ha estado a cargo, en más del 92\%, de las propias instituciones universitarias, complementadas en esta tarea por las asociaciones académicas, algunas dependencias gubernamentales y organizaciones civiles no lucrativas. La participación de editoras comerciales no llega al $8 \%$, porcentaje extremadamente bajo, que requeriría otro tipo de insumos para intentar explicarse, sobre todo por ser constante a lo largo de cinco décadas.

Por otra parte, más del $11 \%$ de la producción total considerada ha sido publicada fuera de México, especialmente en otros países latinoamericanos. La participación de investigadores mexicanos ha sido constante en las actividades y publicaciones de organismos de escala regional, como CIESPAL, FELAFACS y ALAIC, pero también se han desarrollado otros tipos de colaboraciones académicas con instituciones tanto latinoamericanas como europeas o norteamericanas. La circulación propiamente "internacional" de la investigación mexicana de la Comunicación es, sin embargo, a lo largo de las décadas, extremadamente precaria, pues es ínfima la proporción de los productos que han sido publicados en inglés. Por instituciones, llama la atención que los porcentajes más altos de publicaciones en el extranjero correspondan al ITESO (31.1\%), la UdeG (18.3\%) y el ITESM (18.2\%); los intermedios, pero superiores al promedio nacional, a la UAM (13.9\%) y la Universidad de Colima (12.8\%); mientras que los menores a la UIA (10.1\%), la UNAM (5.7\%) y "Otras" (5.3\%).

Es evidente que la distribución de frecuencias y porcentajes de los productos publicados de investigación mexicana sobre la Comunicación, en este trabajo relacionada con una temporalidad de diez lustros, mostrará variaciones en otras escalas temporales. Por ejemplo, volviendo al Cuadro n. 2, si se considera únicamente la producción de los últimos diez años, los porcentajes por institución bajan con respecto al corpus de cinco décadas, en todos los casos excepto en el de la UdeG (que se sitúa en "primer lugar" con el $12.6 \%$ de los productos) y del ITESM (que con el 5.7\% supera a la 
UCOL). Pero sobre todo, aumenta considerablemente el porcentaje de las "Otras" instituciones, al pasar del 30.5\% al 40.0\%, indicando muy probablemente que, independientemente del "estancamiento" general, la "concentración" tiende a reducirse.

Finalmente, se hace indispensable redoblar los esfuerzos por elaborar sistemas de categorías descriptivas suficientemente consistentes para sistematizar las tendencias temático-referenciales y teórico-metodológicas que orientan los productos de la investigación mexicana de la "Comunicación", pues las evidencias de "fragmentación", detectadas en todo el mundo, tendrían que ser analizadas, sobre todo, mediante diseños comparativos, tanto "internos" como inter-nacionales, para los cuales tales sistemas de categorías son condición esencial. En un trabajo reciente (Fuentes, 2010a), se ha probado una categorización temática desarrollada a partir de una propuesta brasileña, que eventualmente podría servir bien para estos fines ${ }^{8}$, y que se condensa en el Cuadro n. 6:

La fragmentación que se ha postulado como tendencia central de los estudios sobre la Comunicación en distintas regiones del mundo (Fuentes, 2010c), tendría que ser reconocida mediante esta $\mathrm{u}$ otras propuestas de categorías temáticas (como la empleada por la Enciclopedia Internacional de Comunicación, Donsbach, ed. 2008, organizada en 29 "áreas editoriales"), pues evidentemente el campo está conformado por múltiples "interdisciplinariedades". Es tarea de la "meta-investigación de la Comunicación" (2007) sistematizar y analizar la producción concreta de investigaciones en diferentes países o instituciones, pues es cada vez más cierto que "en la época actual de extensa especialización, los investigadores tienden a saber poco sobre otros acercamientos, distintos a los propios. Las concepciones de los campos de investigación frecuentemente se basan más en supuestos personales y comunes que en conocimiento producido por análisis empíricos" (Herkman, 2008, p.145).

\footnotetext{
${ }^{8}$ Se trata de un sistema de categorías descriptivas derivado de una clasificación temática en proceso de desarrollo colegiado en Brasil. En su tesis doctoral, Richard Romancini (2006: 157) aplicó una división del campo de la Comunicación en seis "subáreas" temáticas. La adaptación generada consistió en una "ramificación" subsecuente de estas seis "subáreas" en un total de 30 "sub-categorías" (Fuentes, 2010ํㅡㄹ p.28)
} 
Cuadro n. 6: Propuesta de categorías y subcategorías temáticas para productos de investigación de la Comunicación (Fuentes, 2010a)

\begin{tabular}{|c|c|}
\hline Categorías & Sub-categorías \\
\hline \multirow{3}{*}{ Teorías de la Comunicación } & Campo académico \\
\hline & Discursos teóricos \\
\hline & Formación profesional \\
\hline \multirow{5}{*}{ Periodismo y producción editorial } & Agentes Periodísticos \\
\hline & Géneros Periodísticos \\
\hline & Historia de la Prensa \\
\hline & Opinión Pública \\
\hline & Publicaciones periódicas \\
\hline \multirow{5}{*}{ Comunicación Audiovisual } & Cine \\
\hline & Producción Audiovisual \\
\hline & Radio \\
\hline & Televisión \\
\hline & Video \\
\hline \multirow{3}{*}{ Comunicación Institucional } & Comunicación Organizacional \\
\hline & Propaganda \\
\hline & Relaciones Públicas \\
\hline \multirow{4}{*}{ Ciberculturas y Tecnologías de la Comunicación } & Ciberculturas \\
\hline & Producción Multimedia \\
\hline & Telecomunicaciones \\
\hline & Tecnología Educativa \\
\hline \multirow{9}{*}{ Mediaciones e interfaces comunicacionales } & $\begin{array}{l}\text { Industrias Culturales } \\
\text { Análisis del Discurso }\end{array}$ \\
\hline & Comunicación para el desarrollo \\
\hline & Culturas urbanas \\
\hline & Educación \\
\hline & Imaginarios \\
\hline & Legislación \\
\hline & Oferta/Consumo Cultural \\
\hline & Políticas Culturales \\
\hline & Política \\
\hline
\end{tabular}

De las constantes detectadas en análisis comparativos internacionales como el avanzado por el Centro de Investigación de la Comunicación de la Universidad de Helsinki, Finlandia, y los desafíos para el futuro de la investigación (el cambiante paisaje mediático, la orientación nacional/ internacional, la calidad de la investigación, la afirmación del carácter institucional de la disciplina y las relaciones entre la academia y la industria), puede destacarse que: 
La desregulación y liberalización de los medios ha abierto los mercados mediáticos nacionales -al menos en alguna medida- a la competencia internacional o global. La concentración de la propiedad de los medios y las fusiones de empresas mediáticas han sido uno de los resultados de este proceso y han incrementado la dependencia de los mercados nacionales con respecto a las corporaciones mediáticas globales (...). Al mismo tiempo los medios académicos y la investigación de la Comunicación han permanecido atadas a lo nacional, debido a su compromiso con los lenguajes nacionales y las fronteras culturales, así como a las políticas educativas y mediáticas nacionales. Muchos académicos entrevistados vieron una contradicción entre el énfasis en la investigación realizada en perspectiva nacional y el énfasis de la industria por la internacionalización (HERKMAN, 2008, p.156).

El "balance" o articulación de prioridades entre las perspectivas nacional e internacional es, sin duda, una de las cuestiones que con mayor urgencia debieran decidirse en todas partes. Pero también, de esta experiencia finlandesa puede extraerse otra propuesta para la investigación latinoamericana: la conveniencia de partir de "mapeos" empíricos y descriptivos, como los que impulsó la Asociación Latinoamericana de Investigadores de la Comunicación (ALAIC) en los años ochenta, y que dio lugar a la publicación (en muchos casos por primera y lamentablemente única vez) de sistematizaciones documentales de la producción de investigación de la Comunicación por país (Peirano y Kudo, 1982; Munizaga y Rivera, 1983; Marques de Melo, 1984; Anzola y Cooper, 1985; Rivera, 1986; Fuentes, 1988; Beltrán et al, 1990). Tres décadas después, sería una buena idea reeditar el proyecto, en la perspectiva de la internacionalización de la investigación latinoamericana, que no obstante algunos avances (notables en el caso brasileño), permanece en un estado de franco estancamiento.

\section{REFERENCIAS}

ANZOLA, Patricia; PATRÍCIO Cooper. La investigación en comunicación social en Colombia. Lima: DESCO, 1985.

BELTRÁN, Luis Ramiro; SUÁREZ, Carlos; ISAZA, Guillermo. Bibliografía de estudios sobre comunicación en Bolivia. La Paz: PROINSA/IDRC, 1990.

DONSBACH, Wolfgang. (ed.) The International Encyclopedia of Communication. New York: Blackwell, 2008. 
FELAFACS. Mapa de los centros y programas de formación de comunicadores y periodistas en América Latina y el Caribe. Lima: FELAFACS/ UNESCO, 2009.

FUENTES Navarro, Raúl. La investigación de comunicación en México. Sistematización Documental 1956-1986. México: Ediciones de Comunicación, 1988.

. La emergencia de un campo académico: continuidad utópica y estructuración científica de la investigación de la Comunicación en México. Guadalajara: ITESO/ Universidad de Guadalajara, 1998.

- La documentación académica y la producción de conocimiento en ciencias de la Comunicación. Revista Latinoamericana de Ciencias de la Comunicación. São Paulo: Asociación Latinoamericana de Investigadores de la Comunicación, n. 2, p.64-74, 2005.

. Fontes bibliográficas da pesquisa acadêmica nos cursos de pósgraduação em comunicação no Brasil e no México: uma aproximação da análise comparativa. Matrizes. , São Paulo: ECA Universidade de São Paulo: n. 1, p.165-177, 2007.

- Maestrías en Comunicación en México: análisis comparativo de sus perfiles de producción y reproducción académica. Comunicación y Sociedad. Guadalajara: DECS Universidad de Guadalajara, n. 14,p.13-48, 2010a.

- Medio siglo del estudio universitario de la Comunicación en México: el riesgo del inmediatismo superficial. Anuario Coneicc de Investigación de la Comunicación .México: Consejo Nacional para la Enseñanza y la Investigación de las Ciencias de la Comunicación, v. XVII, p.99-115, 2010 b.

. Investigación de la Comunicación: referentes y condiciones internacionales de un diálogo transversal de saberes. Signo y Pensamiento. Bogotá: Pontificia Universidad Javeriana, no. 57, p.38-48,2010c.

HERKMAN, Juha. Current Trends in Media Research. Nordicom Review. Göteborg: Nordicom, v. 29 no. 1., p.145-169, 2008.

MARQUES DE MELO, José (coord.) Inventário da pesquisa em Comunicação no Brasil 1883-1983. São Paulo: INTERCOM/ALAIC, 1984.

MELLADO, Claudia. Análisis estructural de la investigación empírica sobre el periodista latinoamericano. Comunicación y Sociedad. Guadalajara: DECS Universidad de Guadalajara,no. 13, p.125-147, 2010a. 
- La voz de la academia: reflexiones sobre periodismo y Comunicación.

Signo y Pensamiento. Bogotá: Pontificia Universidad Javeriana, n. 56, p.274287, 2010b.

MUNIZAGA, Giselle; RIVERA. La investigación en comunicación social en Chile. Lima: DESCO/Ceneca, 1983.

PEIRANO, Luis; KUDO, Tokihiro. La investigación en comunicación social en el Perú. Lima: DESCO, 1982.

RIVERA, Jorge B. La investigación en comunicación social en Argentina. Lima: DESCO/ASAICC, 1986.

ROMANCINI, Richard. O campo científico da comunicação no Brasil: institucionalização e capital científico, 2006. Tese (Doutorado em Ciências da Comunicação) - Escola de Comunicações e Artes da Universidade de São Paulo (USP).

Recebido em: 25/10/2010 Aceito em: 12/02/2011 